\title{
Pharmaceutical intervention for adverse events improves quality of life in patients with cancer undergoing outpatient chemotherapy
}

Hironori Fujii ${ }^{12^{*}+}$, Yukino Ueda ${ }^{2 \dagger}$, Chiemi Hirose ${ }^{1}, K^{\prime}$ Koichi Ohata ${ }^{1}$, Kumiko Sekiya $^{1}$, Mika Kitahora ${ }^{1}$, Shiori Sadaka ${ }^{1}$, Senri Yamamoto ${ }^{1}$, Daichi Watanabe ${ }^{1}$, Hiroko Kato-Hayashi ${ }^{1}$, Hirotoshi lihara ${ }^{1,2}$, Ryo Kobayashi ${ }^{1}$, Miho Kaburaki ${ }^{3}$, Nobuhisa Matsuhashi ${ }^{4}$, Takao Takahashi ${ }^{4}$, Akitaka Makiyama ${ }^{4}$, Kazuhiro Yoshida ${ }^{4}$, Hideki Hayashi ${ }^{2,3}$ and Akio Suzuki ${ }^{1,2}$

\begin{abstract}
Background: The effect of pharmaceutical intervention to treat adverse events on quality of life (QOL) in outpatients receiving cancer chemotherapy is unclear. We investigated whether pharmaceutical intervention provided by pharmacists in collaboration with physicians improves QOL with outpatient cancer chemotherapy.
\end{abstract}

Methods: We conducted a single-center retrospective descriptive study of pharmaceutical intervention for patients receiving outpatient cancer chemotherapy at Gifu University Hospital between September 2017 and July 2020. We assessed patient QOL using the Japanese version of the EuroQol 5 Dimension5 Level (EQ-5D-5L). Adverse events were graded using the Common Terminology Criteria for Adverse Events (CTCAE) version 4.0. We compared the EQ-5D-5L utility value and incidence of grade 2 or higher adverse events before and after pharmaceutical intervention.

Results: Our analysis included 151 patients who underwent 210 chemotherapy cycles. Pharmaceutical intervention significantly improved patients' EQ-5D-5L utility values from 0.8197 to $0.8603(P<0.01)$. EQ-5D-5L utility values were significantly improved after pharmaceutical intervention for nausea and vomiting (pre-intervention 0.8145 , postintervention $0.8603, P=0.016$ ), peripheral neuropathy (pre-intervention 0.7798 , post-intervention $0.7988, P=0.032$ ) and pain (pre-intervention 0.7625 , post-intervention $0.8197, P=0.035$ ). Although not statistically significant, the incidence of grade 2 or higher adverse events, including nausea and vomiting, dermopathy, pain, oral mucositis, diarrhea and dysgeusia, tended to be lower post-intervention than pre-intervention.

Conclusions: Pharmaceutical intervention by pharmacists in collaboration with physicians may improve QOL in patients undergoing outpatient cancer chemotherapy.

\footnotetext{
* Correspondence: h_fujii@gifu-u.ac.jp

${ }^{\dagger} \mathrm{H}$. Fujii and Y. Ueda contributed equally to this work as first authors.

'Department of Pharmacy, Gifu University Hospital, Yanagido 1-1, Gifu 501-1194, Japan

${ }^{2}$ Laboratory of Pharmacy Practice and Social Science, Gifu Pharmaceutical University, Gifu, Japan

Full list of author information is available at the end of the article
}

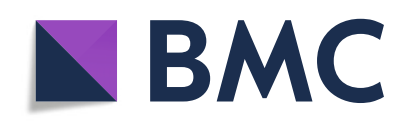

(- The Author(s). 2022 Open Access This article is licensed under a Creative Commons Attribution 4.0 International License, which permits use, sharing, adaptation, distribution and reproduction in any medium or format, as long as you give appropriate credit to the original author(s) and the source, provide a link to the Creative Commons licence, and indicate if changes were made. The images or other third party material in this article are included in the article's Creative Commons licence, unless indicated otherwise in a credit line to the material. If material is not included in the article's Creative Commons licence and your intended use is not permitted by statutory regulation or exceeds the permitted use, you will need to obtain permission directly from the copyright holder. To view a copy of this licence, visit http://creativecommons.org/licenses/by/4.0/. The Creative Commons Public Domain Dedication waiver (http://creativecommons.org/publicdomain/zero/1.0/) applies to the data made available in this article, unless otherwise stated in a credit line to the data. 
Keywords: Quality of life, Pharmaceutical intervention, Adverse events related outpatient cancer chemotherapy, EuroQol 5 Dimension5 level (EQ-5D-5L), Retrospective observational study

\section{Background}

Cancer is a leading cause of death, and cancer incidence is increasing globally, with a corresponding increase in the number of patients receiving cancer chemotherapy $[1,2]$. Historically, anticancer drug therapy was performed only in hospital settings, but advances in the development of supportive therapy and modifications to the healthcare environment to reduce medical costs have facilitated broad-scale outpatient cancer chemotherapy $[3,4]$. Although quality of life $(\mathrm{QOL})$ is higher in patients receiving outpatient cancer chemotherapy than in those treated in hospital [5], the decreased access to medical management places these patients at risk of worse outcomes for several adverse events associated with anticancer drug therapy $[6,7]$.

We previously reported a significant correlation between adverse events, such as peripheral neuropathy, malaise and edema of the limbs, and decreased QOL, regardless of type of cancer or anticancer drug [8]. Similarly, Blanca Prieto-Callejero et al. reported that adverse events, such as nausea, dysgeusia, peripheral neuropathy and loss of appetite have a negative impact on QOL [9]. Iwamoto et al. reported severe mucositis/stomatitis affected health-related QOL in patients treated with cetuximab plus standard chemotherapy for advanced/ metastatic colorectal cancer [10]. Moreover, Mol et al. found that patients with advanced-stage lung cancer who experienced strong negative feelings regarding the side effects of chemotherapy also showed decreased QOL, and recommended that physicians facilitate vigorous management of low-grade adverse events to improve QOL [11]. These reports highlight the necessity of reducing adverse events to enhance QOL. In turn, medical staff, including pharmacists, should therefore conduct monitoring and management for patients receiving outpatient-cancer chemotherapy. Pharmacists have the important role of selecting the appropriate drug therapy and contributing to adverse event reduction and supportive care by communicating with patients receiving cancer chemotherapy through patient interviews [1214]. Pharmaceutical interventions have been shown to improve QOL in outpatients receiving cancer chemotherapy [15-17]. Although some studies have reported that patient education conducted by pharmacists had a positive impact, the effect of pharmaceutical intervention provided in collaboration with physicians to manage adverse events has not been evaluated.

Here, we investigated whether pharmaceutical intervention provided by pharmacists in collaboration with physicians improved QOL in outpatient cancer chemotherapy.

\section{Methods \\ Study design}

This retrospective observational study was conducted at Gifu University Hospital. Patients who underwent outpatient cancer chemotherapy between September 2017 and July 2020 were enrolled. We extracted utility values of QOL, adverse events, pharmaceutical intervention for adverse events, chemotherapy regimens, and other patient demographics from electronic medical records. $\mathrm{Pa}$ tients who underwent pharmaceutical intervention by pharmacists in collaboration with physicians for adverse events were included. Patients with missing or incomplete pre- or post-intervention QOL assessments and patients whose anticancer drugs were reduced by a physician were excluded.

\section{Assessment of QOL}

We used EuroQol 5 Dimension 5 Level (EQ-5D-5L) to assess health-related QOL in patients undergoing outpatient cancer chemotherapy. The EQ-5D-5L questionnaire is a generic health status measure developed by the EuroQol group [18] to calculate quality-adjusted life years [19]. We used the Japanese version of the EQ-5D5L, which was developed by Shiroiwa et al. in 2015 [20].

The questionnaire consists of five dimensions: mobility, self-care, usual activities, pain/discomfort, and anxiety/depression. Each dimension has 5 levels: level 1, no problem; level 2, slight problem; level 3, moderate problem; level 4, severe problem; level 5, unable or extreme [18]. A utility value ranging from 0 to 1 is calculated from the EQ-5D-5L, in which 0 indicates death and 1 indicates full health [21].

Patients who underwent outpatient cancer chemotherapy were asked to answer the EQ-5D-5L questions after each cycle. The Japanese version of the EQ-5D-5L questionnaire was used in face-to-face interviews to estimate the utility values of QOL and was routinely implemented by pharmacists during each patient visit. The utility values were recorded in the hospital's electronic medical records system.

\section{Assessment of adverse events}

In the outpatient chemotherapy clinic, physicians, pharmacists and nurses collaborated to assess adverse events, such as nausea and vomiting, diarrhea, oral mucositis, dysgeusia, peripheral neuropathy, pain, malaise, alopecia, 
and skin disorders. The severity of each adverse event was graded according to the Common Terminology Criteria for Adverse Events (CTCAE) version 5.0 [22]. All patients were provided with a daily checklist to confirm their side effects on their first visit to the outpatient chemotherapy clinic. Using the checklist, patients recorded the occurrence of daily adverse events after chemotherapy. From the returned checklists and the results of the interviews, pharmacists, in collaboration with physicians, recorded the severity of adverse events in the electronic medical records system. Physicians and pharmacists provided pharmaceutical intervention based on clinical practice guidelines to treat moderate or severe adverse events occurring during cancer treatment, and the effect of the intervention on the particular adverse event was assessed at the next consultation.

\section{Effect of pharmaceutical care on adverse events}

We evaluated changes in QOL in patients who developed adverse events at two points, namely the onset of the adverse event (pre-intervention) and after pharmaceutical intervention for the adverse events (post-intervention). The timing of pre-intervention was the patient visit after the onset of each adverse event. The timing of post-intervention was the next patient visit after pharmaceutical intervention.

\section{Statistical analysis}

We used IBM SPSS version 22 (IBM Japan Ltd., Tokyo, Japan) to analyze data. $P$-values $<0.05$ were considered statistically significant. We summarized continuous variables using medians with 25th and 75th percentiles and categorical variables using frequencies and percentages.

The Wilcoxon signed-rank test was conducted to assess the effect of pharmaceutical interventions on QOL in patients experiencing adverse events. We compared the incidence of grade 2 or higher adverse events between pre- and post-intervention using McNemar's test.

\section{Results \\ Patients}

Among patients who received pharmaceutical intervention for adverse events between September 2017 and July 2020, QOL was measured using EQ-5D-5L in 334 cases. Among these, we excluded 84 cases that were exacerbated by adverse events unrelated to the intended intervention, 32 cases whose anticancer drug doses were reduced by a physician, and 8 cases that did not have complete QOL data. We included 210 interventions (151 patients) in the analysis (Fig. 1). Patient characteristics are summarized in Table 1 . The EQ-5D-5L questionnaire was obtained from 151 patients who received pharmaceutical intervention for adverse events. The most common type of cancer was breast cancer (27.8\%), followed by colorectal (26.5\%), pancreatic (13.2\%), gastric (11.3\%), head and neck (6.0\%), and lung (6.0\%). The most common type of regimen was oxaliplatin-based chemotherapy (21.2\%), followed by paclitaxel/nanoparticle albumin-bound paclitaxel-based chemotherapy (17.9\%), anthracyclines + cyclophosphamide (11.3\%), gemcitabine

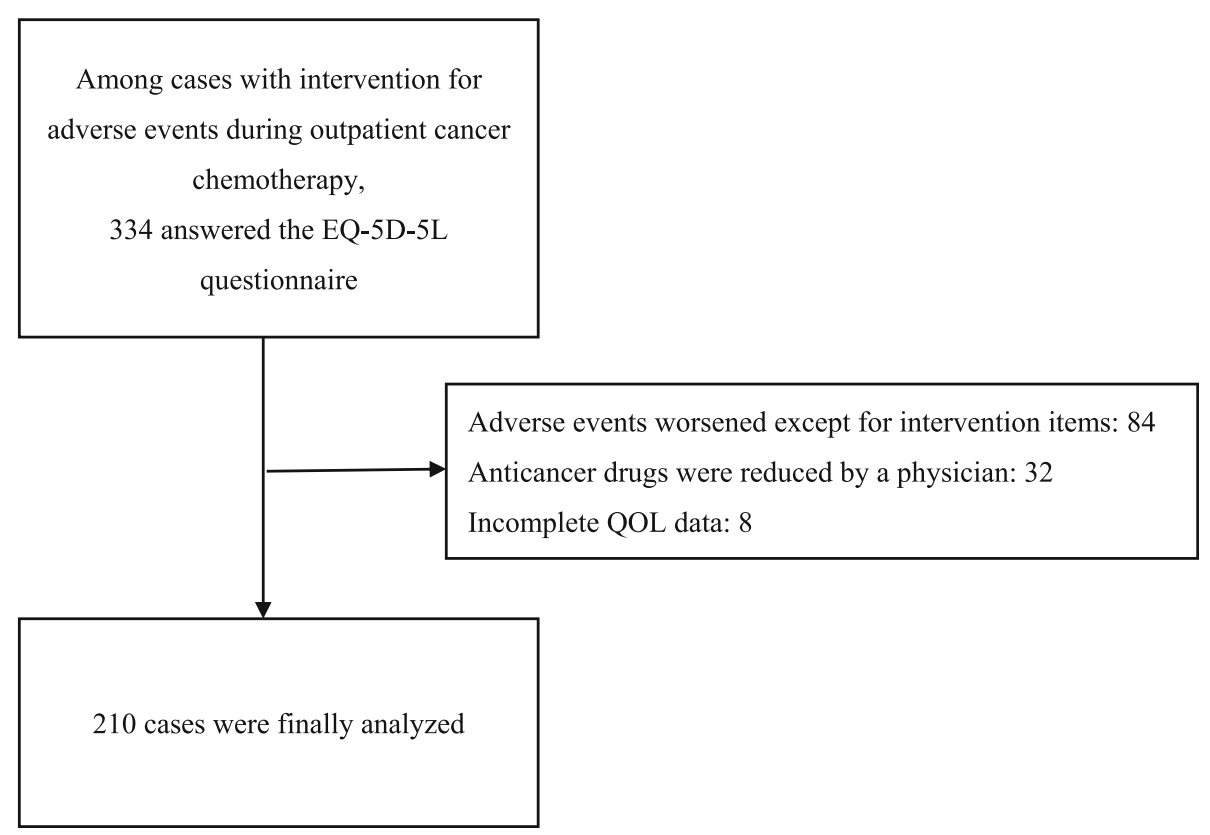

Fig. 1 CONSORT diagram 
Table 1 Patient demographics

\begin{tabular}{|c|c|c|}
\hline Number of patients (male/female) & 151 & $(63 / 88)$ \\
\hline Age, median (min-max) & 60 & $(28-82)$ \\
\hline Number of chemotherapy courses & 210 & \\
\hline \multicolumn{3}{|l|}{ Cancer } \\
\hline Breast cancer & 42 & $27.8 \%$ \\
\hline Colorectal cancer & 40 & $26.5 \%$ \\
\hline Pancreatic cancer & 20 & $13.2 \%$ \\
\hline Gastric cancer & 17 & $11.3 \%$ \\
\hline Head and neck cancer & 9 & $6.0 \%$ \\
\hline Lung cancer & 9 & $6.0 \%$ \\
\hline Ovarian cancer/cervical cancer/uterine cancer & 5 & $3.3 \%$ \\
\hline Biliary tract cancer & 4 & $2.6 \%$ \\
\hline Angiosarcoma & 1 & $0.7 \%$ \\
\hline Esophageal cancer & 1 & $0.7 \%$ \\
\hline Malignant lymphoma & 1 & $0.7 \%$ \\
\hline Malignant pleural mesothelioma & 1 & $0.7 \%$ \\
\hline Peritoneal cancer & 1 & $0.7 \%$ \\
\hline \multicolumn{3}{|l|}{ Regimen } \\
\hline L-OHP + fluoropyrimidines \pm Bmab/Pmab/Tmab & 32 & $21.2 \%$ \\
\hline PTX/Nab-PTX \pm Tmab/ramucirumab/Bmab/Cmab & 27 & $17.9 \%$ \\
\hline Anthracyclines+cyclophosphamide & 17 & $11.3 \%$ \\
\hline GEM+Nab-PTX & 12 & $7.9 \%$ \\
\hline $\begin{array}{l}\text { CPT- } 11 \pm \text { fluoropyrimidines } \pm \text { Bmab/aflibercept/ } \\
\text { ramucirumab/Pmab }\end{array}$ & 11 & $7.3 \%$ \\
\hline DOC/GEM/EPINNR/NabPTX+PER+Tmab & 10 & $6.6 \%$ \\
\hline FOLFIRINOX/FOLFOXIRI+Bmab & 8 & $5.3 \%$ \\
\hline $\mathrm{CBDCA} \pm \mathrm{PEM} \pm \mathrm{Bmab} \pm \mathrm{Cmab}$ & 7 & $4.6 \%$ \\
\hline $\mathrm{GEM} \pm \mathrm{S}-1 \pm \mathrm{CDDP}$ & 7 & $4.6 \%$ \\
\hline Pembrolizumab/nivolumab/durvalmab/atezolizumab & 6 & $4.0 \%$ \\
\hline TAS102 + Bmab & 4 & $2.6 \%$ \\
\hline DOC/LipoDOX \pm Bmab & 3 & $2.0 \%$ \\
\hline Maintenance chemotherapy (Tmab/rituximab) & 2 & $1.3 \%$ \\
\hline$S-1+D O C$ & 2 & $1.3 \%$ \\
\hline Other (CMF/Eribulin/T-DM1) & 3 & $2.0 \%$ \\
\hline
\end{tabular}

L-OHP, oxaliplatin; Bmab, bevacizumab; Pmab, panitumumab; Tmab, trastuzumab; PTX, paclitaxel; Nab-PTX, nanoparticle albumin-bound paclitaxel; Cmab, cetuximab; GEM, gemcitabine; CPT-11, irinotecan; DOC, docetaxel; EPI, epirubicin; VNR, vinorelbine; PER, pertuzumab; FOLFIRINOX/FOLFOXIRI, LOHP + CPT-11 + 5-FU; CBDCA, carboplatin; PEM, pemetrexed; S-1, tegafur+gimeracil+oteracil; CDDP, cisplatin; LipoDOX, doxorubicin liposomal; TAS102, trifluridine; CMF, cyclophosphamide+methotrexate+ 5-FU; T-DM1, trastuzumab emtansine

+ nanoparticle albumin-bound paclitaxel (7.9\%), and irinotecan-based chemotherapy (7.3\%).

\section{Pharmaceutical intervention for adverse events}

The pharmaceutical interventions for adverse events are shown in Table 2. The adverse event with the most interventions was nausea and vomiting (33.1\%; 50/210), followed by peripheral neuropathy $(23.8 \% ; 36 / 210)$, skin disorder $(20.5 \% ; 31 / 210)$, pain $(19.2 \% ; 29 / 210)$, and oral mucositis (16.6\%; 25/210). The most common intervention for nausea and vomiting was oral administration of a D2 receptor blocker (48.0\%), followed by olanzapine (18.0\%), and aprepitant (12.0\%). For peripheral neuropathy, the most common intervention was oral administration of duloxetine (47.2\%) followed by neuropathic pain-alleviating agents (38.9\%), and cryotherapy (11.1\%). External application of steroid cream (38.7\%) was the most common intervention for skin disorders, followed by heparinoids (25.8\%), and oral administration of $\mathrm{H} 1$ receptor blockers $(12.9 \%)$. For pain, the most common intervention was oral administration of non-steroidal anti-inflammatory drugs $(44.8 \%)$, followed by opioids (37.9\%), and acetaminophen (24.1\%).

\section{Relationship between pharmaceutical intervention and EQ-5D-5L utility values}

The median EQ-5D-5L utility value and proportion of patients scoring at level 2 or higher in each dimension pre- and post-intervention are shown in Table 3. Overall, EQ-5D-5L utility values significantly improved $(0.8197$ pre to 0.8603 post; $P<0.01)$ after pharmaceutical intervention for adverse events. A significant change in the proportion of patients with level 2 or higher scores pre- and post-intervention was observed only in the 'mobility' dimension ( $37.1 \%$ pre to $30.5 \%$ post; $P=0.014$ ).

\section{Pre- and post-intervention changes in EQ-5D-5L utility} values and incidence of grade 2 or higher adverse events Figure 2 shows the median EQ-5D-5L utility values preand post-intervention for each adverse event. EQ-5D-5L utility values significantly improved from pre- to postintervention for nausea and vomiting (0.8145 pre to 0.8603 post; $P=0.016)$, peripheral neuropathy $(0.7798$ pre to 0.7988 post; $P=0.032)$, and pain $(0.7625$ pre to 0.8197 post; $P=0.035)$. Although not statistically significant, the median EQ-5D-5L utility values tended to increase for skin disorder (0.8603 pre to 0.8978 post; $P=0.057)$, oral mucositis $(0.8603$ pre to 0.8978 post; $P=0.121)$ and diarrhea $(0.7923$ pre to 0.8197 post; $P=0.328)$. Comparing the proportion of level 2 or higher scores in the 5 dimensions for each adverse event, there were no significant changes pre- and post-intervention (data not shown).

The incidence of adverse events is shown in Fig. 3. The incidence of grade 2 or higher nausea and vomiting (56.0\% pre to $40.0 \%$ post; $P=0.18)$, skin disorder $(22.6 \%$ pre to $9.7 \%$ post; $P=0.125)$, pain $(31.0 \%$ pre to $20.7 \%$ post; $P=1.0)$, oral mucositis $(36.0 \%$ pre to $16.0 \%$ post; $P=0.25)$, diarrhea (33.4\% pre to $20.0 \%$ post; $P=1.0$ ) and dysgeusia (58.3\% pre to $33.3 \%$ post; $P=0.375$ ) tended to 
Table 2 Pharmaceutical intervention for adverse events

\begin{tabular}{|c|c|c|c|}
\hline Adverse event & Intervention & Number & Rate \\
\hline \multirow[t]{7}{*}{ Nausea and vomiting $(n=50)$} & D2 blocker & 24 & $48.0 \%$ \\
\hline & Olanzapine & 9 & $18.0 \%$ \\
\hline & Aprepitant & 6 & $12.0 \%$ \\
\hline & 5-HT3 receptor antagonist & 4 & $8.0 \%$ \\
\hline & Proton-pump inhibitor (PPI) & 3 & $6.0 \%$ \\
\hline & Mirtazapine & 2 & $4.0 \%$ \\
\hline & Others (Camostat/Butylscopolamine/Dexamethasone) & 3 & $6.0 \%$ \\
\hline \multirow[t]{4}{*}{ Peripheral neuropathy $(n=36)$} & Duloxetine & 17 & $47.2 \%$ \\
\hline & Pregabalin/Mirogabalin Cryotherapy & 14 & $38.9 \%$ \\
\hline & Dose reduction of oxaliplatin (from $85 \mathrm{mg} / \mathrm{m} 2$ to $65 \mathrm{mg} / \mathrm{m} 2$ ) & 4 & $11.1 \%$ \\
\hline & & 1 & $2.8 \%$ \\
\hline \multirow[t]{6}{*}{ Skin disorder $(n=31)$} & Steroid cream & 12 & $38.7 \%$ \\
\hline & Heparinoids & 8 & $25.8 \%$ \\
\hline & $\mathrm{H} 1$ receptor blocker & 4 & $12.9 \%$ \\
\hline & Antimicrobial agent & 3 & $9.7 \%$ \\
\hline & Pemirolast & 3 & $9.7 \%$ \\
\hline & Others (Posterisan ${ }^{\circledR}$ forte/Urea cream/Crotamiton) & 3 & $9.7 \%$ \\
\hline \multirow[t]{4}{*}{ Pain $(n=29)$} & Non-steroidal anti-inflammatory drugs (NSAIDs) & 13 & $44.8 \%$ \\
\hline & Opioids & 11 & $37.9 \%$ \\
\hline & Acetaminophen & 7 & $24.1 \%$ \\
\hline & Shakuyakukanzoto ${ }^{a}$ & 1 & $3.4 \%$ \\
\hline \multirow[t]{4}{*}{ Oral mucositis $(n=25)$} & Sodium azulenesulfonate preparation & 15 & $60.0 \%$ \\
\hline & Steroid & 7 & $28.0 \%$ \\
\hline & Sodium alginate & 2 & $8.0 \%$ \\
\hline & Zinc preparation & 2 & $8.0 \%$ \\
\hline \multirow[t]{5}{*}{ Diarrhea $(n=15)$} & Probiotics & 9 & $60.0 \%$ \\
\hline & Loperamide & 4 & $26.7 \%$ \\
\hline & Hangeshashinto & 3 & $20.0 \%$ \\
\hline & Aluminium silicate & 2 & $13.3 \%$ \\
\hline & Others (Butylscopolamine/Alubumin tannate) & 2 & $13.3 \%$ \\
\hline Dysgeusia $(n=12)$ & Zinc preparation & 12 & $100.0 \%$ \\
\hline \multirow[t]{2}{*}{ Malaise $(n=6)$} & Tapering of dexamethasone & 5 & $83.3 \%$ \\
\hline & Hochuekkito $^{a}$ & 1 & $16.7 \%$ \\
\hline \multirow[t]{3}{*}{ Edema limbs $(n=4)$} & Goreisan $^{a}$ & 2 & $50.0 \%$ \\
\hline & Azosemide & 1 & $25.0 \%$ \\
\hline & Change from pregabalin to duloxetine & 1 & $25.0 \%$ \\
\hline \multirow[t]{2}{*}{ Constipation $(n=2)$} & Probiotics & 1 & $50.0 \%$ \\
\hline & Sennoside & 1 & $50.0 \%$ \\
\hline
\end{tabular}

${ }^{a}$ Shakuyakukanzoto and Hochuekkito are traditional herbal medicines commonly used in North-East Asian countries

be lower post-intervention, albeit that these differences were not significant.

\section{Discussion}

In this study, we investigated the effects of pharmaceutical interventions for adverse events provided by pharmacists in collaboration with physicians on QOL in patients receiving outpatient cancer chemotherapy. Evaluation by EQ-5D-5L revealed that interventions were associated with a significant post-intervention improvement in patient QOL. In particular, interventions for nausea and vomiting, pain, and peripheral 
Table 3 Comparison of EuroQol 5 Dimension-5 Level (EQ-5D-5 L) utility values between pre- and post-intervention

\begin{tabular}{llll}
\hline & Pre-intervention & Post-intervention & $P$ value \\
\hline Utility value (IQR) & $0.8197(0.7096-0.8978)$ & $0.8603(0.7543-0.9384)$ & $<0.01$ \\
Dimension & Proportion of level $\geq 2(\%)$ & & 0.014 \\
Mobility & 37.1 & 30.5 & 0.804 \\
Personal care & 14.3 & 13.3 & 0.185 \\
Usual activities & 41.9 & 38.1 & 0.071 \\
Pain/discomfort & 65.2 & 58.6 & 0.144 \\
Anxiety/depression & 45.7 & 41.0 & \\
\hline
\end{tabular}

210 cases were analyzed. The Wilcoxon signed-rank test was used to compare the EQ-5D-5L utility value before and after pharmaceutical care. The McNemar test was used to compare percentage of level $\geq 2$ in each of 5 dimensions between pre-intervention and post-intervention

neuropathy were associated with significant improvements in QOL before and after the intervention. These results indicate that pharmaceutical intervention provided by pharmacists in collaboration with physicians for adverse events is useful for improving QOL in cancer chemotherapy.

Comparison of EQ-5D-5L utility values before and after pharmaceutical intervention for each of the $5 \mathrm{di}$ mensions revealed that mobility was significantly improved post-intervention. The proportion of patients with a mobility level higher than 2 significantly decreased after pharmaceutical intervention. Similarly, a population-based cohort study of women diagnosed with breast cancer reported that patients with higher exercise metabolic equivalent scores had higher total QOL scores [23]. Eyl et al. also reported that QOL in long-term colorectal cancer survivors was higher in those who were more physically active than non-active survivors [24]. These findings indicate that improved mobility after the receipt of pharmaceutical intervention may contributed to enhancing QOL.

EQ-5D-5L utility values significantly increased after pharmaceutical intervention for nausea and vomiting. Hagiwara et al. showed that grade 1 nausea negatively impacted patient QOL [25]. Chemotherapy-induced nausea and vomiting are common side effects that can greatly impact patients receiving outpatient cancer-chemotherapy [26]. Additionally, the Clinical Practice Guidelines for Antiemesis from the Japan Society of Clinical Oncology highlight the importance of managing delayed nausea and vomiting during outpatient cancer-chemotherapy [27]. At Gifu University Hospital, pharmacists work in collaboration

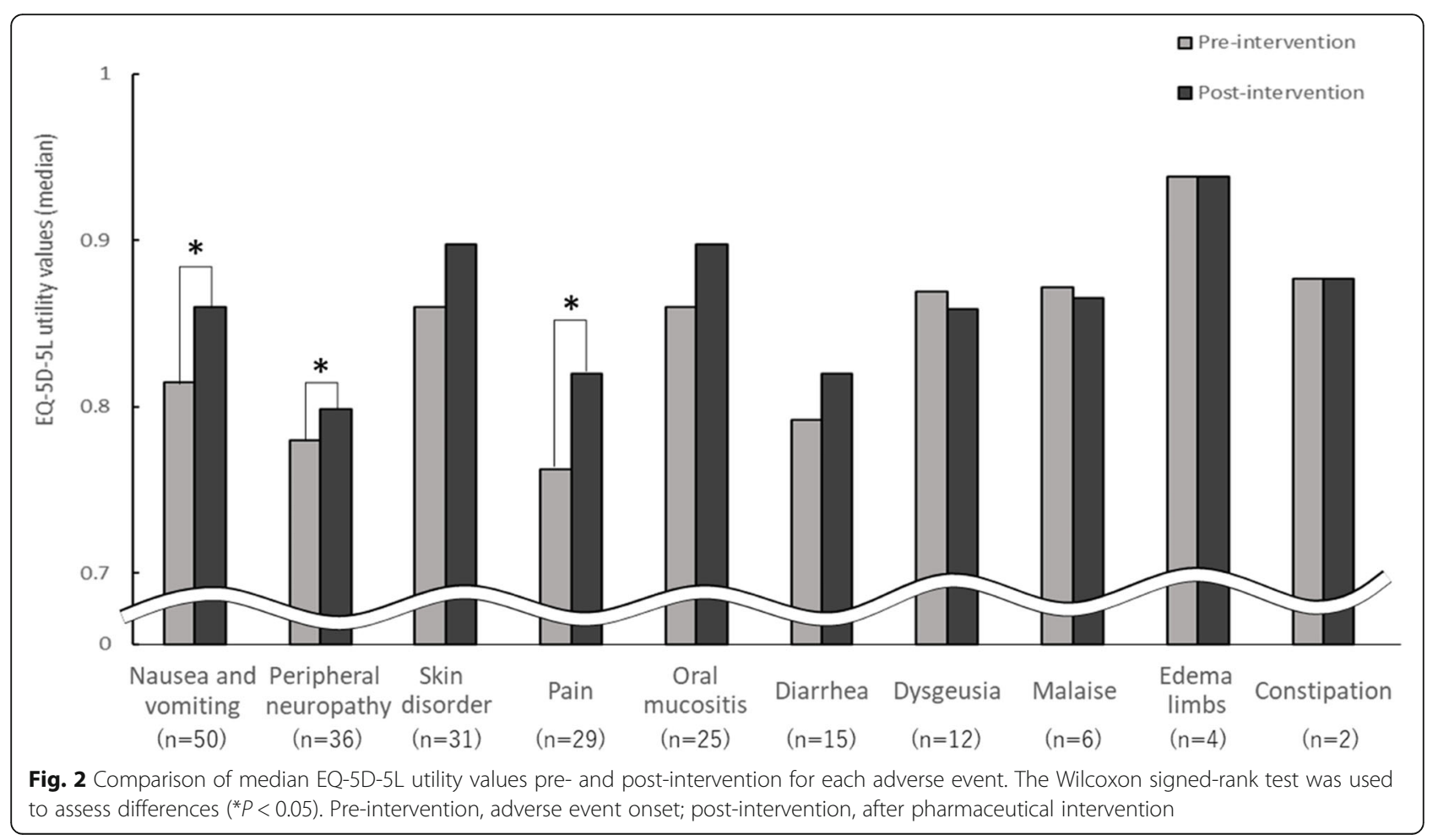




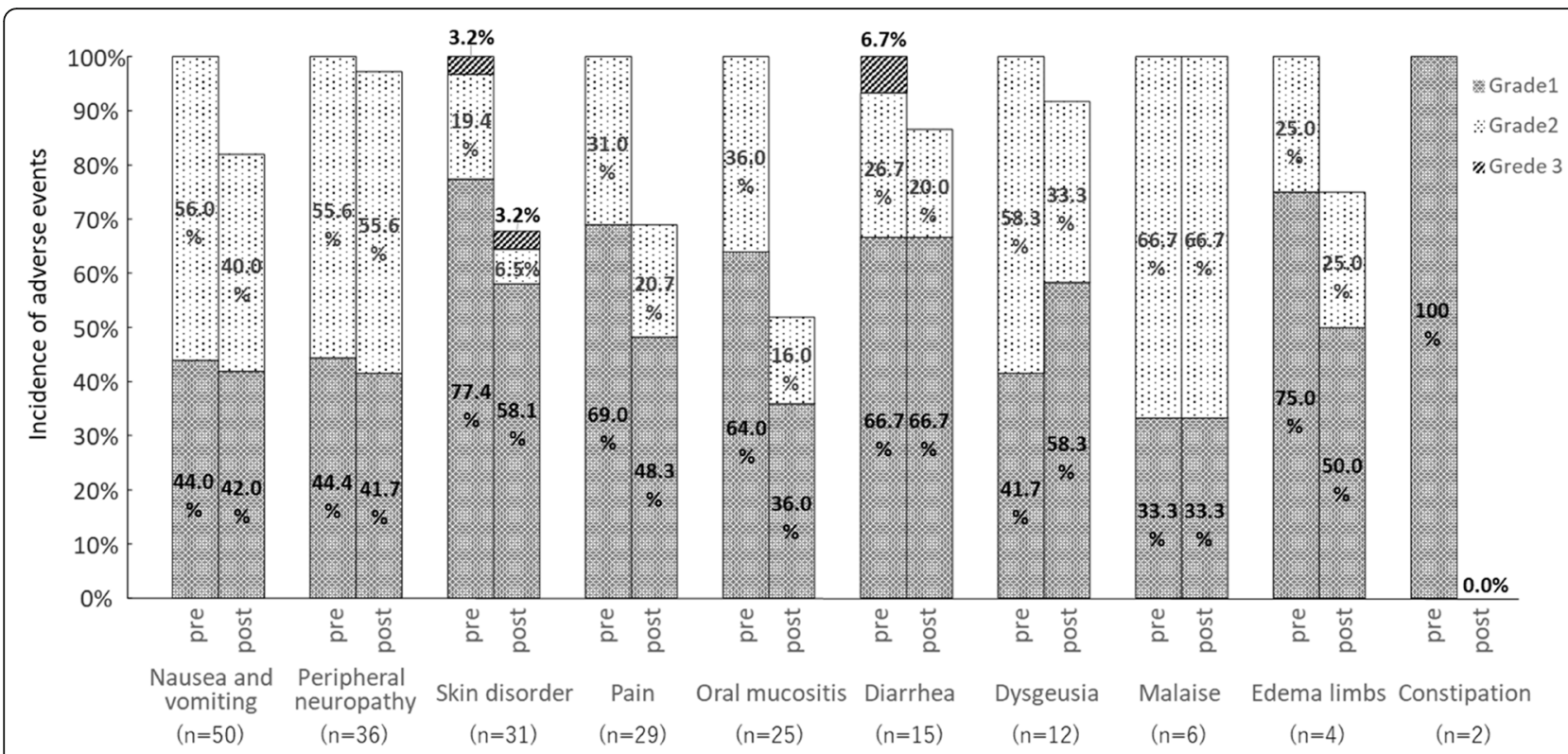

Fig. 3 Comparison of adverse event incidence and grade pre- and post-intervention. McNemar's test was used to compare the incidence of grade 2 or higher adverse events before and after pharmaceutical intervention

with physicians to select antiemetic agents in consideration of comorbidities and the appearance of nausea and vomiting after chemotherapy. Andrea et al. reported that antiemetic responses and patient satisfaction were significantly improved with the use of supportive medication prescribed according to evidence-based treatment guidelines and patient counseling for the management of treatmentassociated adverse events [28]. Thus, individualized antiemetic treatment may contribute to improving patient QOL. Moreover, we found that EQ-5D-5L utility values were significantly improved when aprepitant was orally administered to treat nausea and vomiting (data not shown). Similarly, Thomas et al. reported that the addition of aprepitant to a standard regimen resulted in significantly less chemotherapy-induced nausea and vomiting and improved QOL [29].

Pharmaceutical interventions for pain also significantly improved EQ-5D-5L values. In particular, post-intervention EQ-5D-5L utility values significantly improved after opioids were added to the pain treatment regimen (data not shown). This finding is consistent with previous reports that QOL was improved after oral administration of opioids for severe pain in patients with cancer [30]. Clinical guidelines for cancer pain management suggest that opioids should be administered to cancer patients with moderateto-severe pain [31-34]. The relationship between QOL and pain is inversely proportional in patients with breast cancer [35]. Therefore, the oral administration of opioids can relieve more severe pain and significantly improved patient QOL.
EQ-5D-5L utility values were also significantly improved after pharmaceutical intervention for peripheral neuropathy. Administration of pregabalin and duloxetine $[36,37]$ and the use of frozen gloves (cryotherapy) [38] are reported to improve QOL in patients with peripheral neuropathy. Although overall EQ-5D-5L utility values improved post-intervention, no significant differences were observed in the incidence of grade 2 or higher adverse events. According to the CTCAE, grade 2 peripheral neuropathy is a symptom that affects daily life, and the range of symptoms included in this classification is wide. In terms of the CTCAE assessment of peripheral neuropathy, pharmaceutical interventions were found to improve symptoms, which may have contributed to enhancing patient QOL. To assess the effect of pharmaceutical intervention for peripheral neuropathy, we considered that it would be necessary to use not only CTCAE but also NRS; however, we did not assess peripheral neuropathy using NRS or VAS in this study, and determining which scale is more appropriate for assessment of peripheral neuropathy is a question for future research.

In this study, we demonstrated that pharmaceutical intervention provided by pharmacists in collaboration with physicians contributed to QOL enhancement. Comprehensive pharmaceutical care increases patients' chemotherapy-related knowledge, improves positive emotions, facilitates the management of chemotherapyinduced adverse events, and improves QOL [39]. An association between lower QOL and increased all-cause 
mortality has been reported [40]. Accordingly, to improve therapeutic effects, QOL should be maintained or enhanced in patients undergoing outpatient chemotherapy. The relationship between QOL enhancement and therapeutic effects warrants elucidation in future study.

Several limitations of this study should be acknowledged. First, because we were unable to compare our results with a control group that did not receive pharmaceutical intervention, it is unclear whether the improvement in QOL was due to pharmaceutical intervention or time. Second, the degree of cancer progression and the environment surrounding patients receiving outpatient-cancer chemotherapy can strongly impact QOL. We did not give sufficient consideration to patient condition, such as tumor metastasis, line of treatment, employment, and marital status. Third, it was not possible to clarify confounding factors such as differences among non-physician staff in charge (e.g., whether they were certified nurses or not). Furthermore, this study was a single-center study with a small sample size, which could result in exposure of the study population to a certain common bias. Further studies with a larger sample size are needed to verify our findings.

\section{Conclusion}

This study suggests that the enhancement of supportive care for adverse events, such as nausea and vomiting, peripheral neuropathy, and pain, may improve the QOL of outpatients undergoing chemotherapy. Pharmaceutical intervention provided by pharmacists in collaboration with physicians is useful in maintaining and improving QOL and in the management of adverse events during cancer treatment.

\section{Acknowledgements}

Not applicable.

\section{Authors' contributions}

Conceptualization, H.F.; methodology, H.F.; formal analysis, H.F. and Y.U. investigation, H.F., Y.U.; data curation, C.H., K.O., K.S., M.K., S.S., S.Y., D.W., H.K.H., H.I., and M.K.; writing-original draft preparation, H.F. and Y.U.; Writing-review and editing, H.F., Y.U., H.I., R.K., N.M., T.T., A.M., K.Y., H.H. and A.S.; supervision, A.S. All authors have read and agreed to the published version of the manuscript. The author(s) read and approved the final manuscript.

\section{Funding}

This study did not receive funding from any funding source.

\section{Availability of data and materials}

Not applicable.

\section{Declarations}

\section{Ethics approval and consent to participate}

This retrospective study involving human participants was in accordance with the ethical standards of the institutional and national research committee and with the 1964 Helsinki Declaration and its later amendments or comparable ethical standards. Ethical approval for the study was obtained from the ethics committees of Gifu Pharmaceutical University (Approval
Number: 3-9) and Gifu University Graduate School of Medicine (Approval number: 2021-03).

\section{Consent for publication}

Not applicable.

\section{Competing interests}

K. Yoshida has received honoraria for lectures from Chugai Pharmaceutical Co., Ltd., Taiho Pharmaceutical Co., Ltd., Takeda Pharmaceutical Co., Ltd., Eli Lilly and Company, Yakult Honsha Co., Ltd., Merck Sharp \& Dohme Co., Ltd., Daiichi Sankyo Co., Ltd., Ono Pharmaceutical Co., Ltd., Merck Serono Co., Ltd., Johnson \& Johnson Co., Ltd., Covidien Co., Ltd., Eisai Co., Ltd., Otsuka

Pharmaceutical Co., Ltd., Sanofi K.K., Nippon Kayaku Co., Ltd., Asahi Kasei Co., Ltd., Tsumura Co., Ltd., EA Pharma Co., Ltd., Bayer Yakuhin Co., Ltd., Olympus Co., Ltd., Terumo Co., Ltd., Bristol-Myers Squibb Co., Ltd., Denka Co., Ltd., Teijin Co., Ltd., SBI Pharmaceuticals Co., Ltd., Intuitive Surgical Co., Ltd., Novartis Pharma K.K., and Pfizer Inc.; and research funding from Chugai Pharmaceutical Co., Ltd., Taiho Pharmaceutical Co., Ltd., Takeda Pharmaceutical Co., Ltd., Eli Lilly and Company, Yakult Honsha Co., Ltd., Merck Sharp \& Dohme Co., Ltd., Daiichi Sankyo Co., Ltd., Ono Pharmaceutical Co., Ltd., Merck Serono Co., Ltd., Johnson \& Johnson Co., Ltd., Covidien Co., Ltd., Eisai Co., Ltd., Otsuka Pharmaceutical Co., Ltd., Sanofi K.K., Nippon Kayaku Co., Ltd., Asahi Kasei Co., Ltd., Tsumura Co., Ltd., Kyowa Hakko Kirin Co., Ltd., Astellas Pharma Co., Ltd., Toyama Chemical Co., Ltd., Kinetic Concepts Co., Ltd., Abbott Japan Co., Ltd., and Toray Industries, Co., Ltd. outside the submitted work.

T. Takahashi has received honoraria for lectures from Takeda Pharmaceutical Co., Ltd.

A. Makiyama has received honoraria for lectures from Eli Lilly and Company, Taiho Pharmaceutical Co., Ltd. and Takeda Pharmaceutical Co., Ltd.

The other authors have no conflicts of interest.

\section{Author details}

${ }^{1}$ Department of Pharmacy, Gifu University Hospital, Yanagido 1-1, Gifu 501-1194, Japan. ${ }^{2}$ Laboratory of Pharmacy Practice and Social Science, Gifu Pharmaceutical University, Gifu, Japan. ${ }^{3}$ Laboratory of Community Healthcare Pharmacy, Gifu Pharmaceutical University, Gifu, Japan. ${ }^{4}$ Department of Surgical Oncology, Gifu University Graduate School of Medicine, Gifu, Japan.

Received: 28 December 2021 Accepted: 8 February 2022

Published online: 02 March 2022

\section{References}

1. Sung H, Ferlay J, Siegel RL, Laversanne M, Soerjomataram I, Jemal A, et al. Global Cancer Statistics 2020: GLOBOCAN estimates of incidence and mortality worldwide for 36 cancers in 185 countries. CA Cancer J Clin. 2021; 71(3):209-49. https://doi.org/10.3322/caac.21660

2. Torre LA, Siegel RL, Ward EM, Jemal A. Global Cancer incidence and mortality rates and trends —an update. Cancer Epidemiol Biomark Prev. 2016;25(1):16-27. https://doi.org/10.1158/1055-9965.EPI-15-0578.

3. Gradishar WJ, Tjulandin S, Davidson N, Shaw H, Desai N, Bhar P, et al. Phase III trial of nanoparticle albumin-bound paclitaxel compared with polyethylated castor oil-based paclitaxel in women with breast cancer. J Clin Oncol. 2005;23(31):7794-803. https://doi.org/10.1200/JCO.2005.04.937.

4. Saito M, Aogi K, Sekine I, Yoshizawa H, Yanagita Y, Sakai H, et al. Palonosetron plus dexamethasone versus granisetron plus dexamethasone for prevention of nausea and vomiting during chemotherapy: a doubleblind, double-dummy, randomised, comparative phase III trial. Lancet Oncol. 2009;10(2):115-24. https://doi.org/10.1016/S1470-2045(08)70313-9.

5. Ishiura $Y$, Terasaki $Y$, Yamamoto H, Yokawa S, Fukushima W, Hirosawa $H$, et al. Analysis of factors associated with quality of life for patients with nonsmall lung cancer receiving outpatient vinorelbine therapy as alternative inpatient therapy. Gan To Kagaku Ryoho. 2007;34(9):1401-4.

6. Miyake T, Hashida T, Kobayashi M, Terada T, Katsura T, Yasuda H, et al. Survey on use of Amrubicin hydrochloride in outpatients with lung Cancer and evaluation of its hematologic toxicity. Jpn J Pharm Health Care Sci. 2008:34:268-73.

7. Tsai SC, Liu LN, Tang ST, Chen JC, Chen ML. Cancer pain as the presenting problem in emergency departments: incidence and related factors. Support Care Cancer. 2010;18(1):57-65. https://doi.org/10.1007/s00520-009-0630-6.

8. Hirose C, Fujii H, lihara H, Ishihara M, Nawa-Nishigaki M, Kato-Hayashi H, et al. Real-world data of the association between quality of life using the 
EuroQol 5 dimension 5 level utility value and adverse events for outpatient cancer chemotherapy. Support Care Cancer. 2020;28(12):5943-52. https://doi.org/10.1007/s00520-020-05443-8.

9. Prieto-Callejero B, Rivera F, Fagundo-Rivera J, Romero A, Romero-Martín M, Gómez-Salgado J, et al. Relationship between chemotherapy-induced adverse reactions and health-related quality of life in patients with breast cancer. Med (Baltimore). 2020;99(33):e21695. https://doi.org/10.1097/MD. 0000000000021695.

10. Iwamoto S, Ooki A, Morita S, Hara H, Tanioka H, Satake H, et al. A prospective phase II study to examine the relationship between quality of life and adverse events of first-line chemotherapy plus cetuximab in patients with KRAS wild-type unresectable metastatic colorectal cancer: QUACK trial. Cancer Med. 2018;7(9):4217-27. https://doi.org/10.1002/cam4.1623.

11. de Mol M, Visser S, den Oudsten BL, Lodder P, van Walree N, Belderbos $H$, et al. Frequency of low-grade adverse events and quality of life during chemotherapy determine patients' judgement about treatment in advanced-stage thoracic cancer. Support Care Cancer. 2019;27(9):3563-72. https://doi.org/10.1007/s00520-019-4659-x.

12. Mae $Y$, Yokokawa T, Kawakami $K$, et al. Usefulness of pharmaceutical outpatient clinic in XELOX therapy. Jpn J Pharm Health Care Sci. 2011;37: $611-5$.

13. Sudou M, Morii H, Sakanaka M, Noda S, Wakasugi Y, Hukutake N, et al. Introduction of pharmaceutical care and its effects in outpatients with cancer chemotherapy. Jpn J Pharm Health Care Sci. 2013;39:77-84.

14. Colombo LRP, Aguiar PM, Lima TM, Storpirtis $S$. The effects of pharmacist interventions on adult outpatients with cancer: a systematic review. J Clin Pharm Ther. 2017:42(4):414-24. https://doi.org/10.1111/jcpt.12562.

15. Tanaka K, Hori A, Tachi T, Osawa T, Nagaya K, Makino T, et al. Impact of pharmacist counseling on reducing instances of adverse events that can affect the quality of life of chemotherapy outpatients with breast Cancer. J Pharm Health Care Sci. 2018;4(1):9. https://doi.org/10.1186/s4 0780-018-0105-3.

16. Farrag DK, Sabri NA, Tawfik AS, Shaheen SM. Evaluation of the clinical effect of pharmacist intervention. Eur J Oncol Pharm. 2020;3(1):1. https://doi.org/1 0.1097/OP9.0000000000000023.

17. Periasamy U, Mohd-Sidik S, Akhtari-Zavare M, Rampal L, Ismail SIF, Mahmud R. Effects of counselling on quality of life among Cancer patients in Malaysia: a randomized controlled trial. Iran J Public Health. 2020:49(10): 1902-11. https://doi.org/10.18502/ijph.v49i10.4693.

18. Herdman M, Gudex C, Lloyd A, Janssen M, Kind P, Parkin D, et al. Development and preliminary testing of the new five-level version of EQ-5D (EQ-5D-5L). Qual Life Res. 2011;20(10):1727-36. https://doi.org/10.1007/s1113 6-011-9903-X.

19. Brauer CA, Rosen AB, Greenberg D, Neumann PJ. Trends in the measurement of health utilities in published cost-utility analyses. Value Health. 2006;9(4):213-8. https://doi.org/10.1111/j.1524-4733.2006. 00116.x.

20. Ikeda S, Shiroiwa T, Igarashi A. Developing a Japanese version of the EQ-5D5L value set. J Natl Inst Public Health. 2015;64:47-55.

21. Okamoto T, Hashimoto K, Ohashi M, Nakachi T, Ishii A, Miyano S, et al. Survey on health-related quality of life (HRQOL) and cost-effectiveness for rehabilitation hospital inpatients by EuroQOL. Jpn J Rehabil Medcine. 2004; 41:678-85.

22. US Department of Health and Human Services, National Institutes of Health National Cancer Institute Common terminology criteria for adverse events (CTCAE) version 5.0. J Pharm Health Care Sci. 2021;7(1):27. Available: https:// ctep.cancer.gov/protocoldevelopment/electronic_applications/docs/CTCAE_ v5_Quick_Reference_5x7.pdf. Accessed 21 Jan 2022.

23. Chen $X$, Zheng Y, Zheng W, Gu K, Chen Z, Lu W, et al. The effect of regular exercise on quality of life among breast cancer survivors. Am J Epidemiol. 2009;170(7):854-62. https://doi.org/10.1093/aje/kwp209.

24. Health Qual Life Outcomes. 2018;16:112. Quality of life and physical activity in long-term ( $\geq 5$ years post-diagnosis) colorectal cancer survivors systematic review. Health Qual Life Outcome. 2018;16:112.

25. Hagiwara Y, Shiroiwa T, Shimozuma K, Kawahara T, Uemura Y, Watanabe T, et al. Impact of adverse events on health utility and health-related quality of life in patients receiving first-line chemotherapy for metastatic breast Cancer: results from the SELECT BC study. Pharmacoeconomics. 2018;36(2): 215-23. https://doi.org/10.1007/s40273-017-0580-7.

26. Ihbe-Heffinger $A$, Ehlken $B$, Bernard $R$, Berger $K$, Peschel $C$, Eichler $\mathrm{HG}$, et al. The impact of delayed chemotherapy-induced nausea and vomiting on patients, health resource utilization and costs in German cancer centers. Ann Oncol. 2004;15(3):526-36. https://doi.org/10.1093/annonc/mdh110.

27. Aogi K, Takeuchi H, Saeki T, Aiba K, Tamura K, lino K, et al. Optimizing antiemetic treatment for chemotherapy-induced nausea and vomiting in Japan: update summary of the 2015 Japan Society of Clinical Oncology Clinical Practice Guidelines for Antiemesis. Int J Clin Oncol. 2021;26(1):1-17. https://doi.org/10.1007/s10147-020-01818-3.

28. Liekweg A, Westfeld M, Braun M, Zivanovic O, Schink T, Kuhn W, et al. Pharmaceutical care for patients with breast and ovarian cancer. Support Care Cancer. 2012;20(11):2669-77. https://doi.org/10.1007/s00520-012-1385-z.

29. Schmitt T, Goldschmidt H, Neben K, Freiberger A, Hüsing J, Gronkowski M, et al. Aprepitant, granisetron, and dexamethasone for prevention of chemotherapy-induced nausea and vomiting after high-dose melphalan in autologous transplantation for multiple myeloma: results of a randomized, placebo-controlled phase III trial. J Clin Oncol. 2014;32(30):3413-20. https:// doi.org/10.1200/JCO.2013.55.0095

30. Leppert W, Nosek K. Comparison of the quality of life of Cancer patients with pain treated with Oral controlled-release morphine and oxycodone and transdermal buprenorphine and fentanyl. Curr Pharm Des. 2019;25(30): 3216-24. https://doi.org/10.2174/1381612825666190717091230.

31. Marinangeli F, Ciccozzi A, Leonardis M, Aloisio L, Mazzei A, Paladini A, et al. Use of strong opioids in advanced cancer pain: a randomized trial. J Pain Symptom Manag. 2004;27(5):409-16. https://doi.org/10.1016/j.jpainsymma n.2003.10.006.

32. Maltoni M, Scarpi E, Modonesi C, Passardi A, Calpona S, Turriziani A, et al. A validation study of the WHO analgesic ladder: a two-step vs three-step strategy. Support Care Cancer. 2005;13(11):888-94. https://doi.org/10.1007/ s00520-005-0807-6.

33. Bandieri E, Romero M, Ripamonti Cl, Artioli F, Sichetti D, Fanizza C, et al. Randomized Trial of Low-Dose Morphine Versus Weak Opioids in Moderate Cancer Pain. J Clin Oncol. 2016;34:436-42.

34. Portenoy RK. Treatment of cancer pain. Lancet. 2011;377(9784):2236-47. https://doi.org/10.1016/S0140-6736(11)60236-5.

35. Costa WA, Monteiro MN, Queiroz JF, Gonçalves AK. Pain and quality of life in breast cancer patients. Clin (Sao Paulo). 2017;72(12):758-63. https://doi. org/10.6061/clinics/2017(12)07.

36. Avan R, Janbabaei G, Hendouei N, Alipour A, Borhani S, Tabrizi N, et al. The effect of pregabalin and duloxetine treatment on quality of life of breast cancer patients with taxane-induced sensory neuropathy: a randomized clinical trial. J Res Med Sci. 2018;23(1):52. https://doi.org/10.4103/jrms. JRMS 1068 17.

37. Smith EM, Pang H, Cirrincione C, Fleishman S, Paskett ED, Ahles T, et al. Effect of duloxetine on pain, function, and quality of life among patients with chemotherapy-induced painful peripheral neuropathy: a randomized clinical trial. JAMA. 2013;309(13):1359-67. https://doi.org/10.1001/jama.2 013.2813 .

38. Beijers AJM, Bonhof CS, Mols F, Ophorst J, de Vos-Geelen J, Jacobs EMG, et al. Multicenter randomized controlled trial to evaluate the efficacy and tolerability of frozen gloves for the prevention of chemotherapy-induced peripheral neuropathy. Ann Oncol. 2020;31(1):131-6. https://doi.org/10.1016/ j.annonc.2019.09.006.

39. Wang $Y, W u H, X U F$. Impact of clinical pharmacy services on KAP and QOL in Cancer patients: a single-center experience. Biomed Res Int. 2015;2015: 502431-8. https://doi.org/10.1155/2015/502431.

40. Ratjen I, Schafmayer C, Enderle J, di Giuseppe R, Waniek S, Koch M, et al. Health-related quality of life in long-term survivors of colorectal cancer and its association with all-cause mortality: a German cohort study. BMC Cancer. 2018;18(1):1156. https://doi.org/10.1186/s12885-018-5075-1.

\section{Publisher's Note}

Springer Nature remains neutral with regard to jurisdictional claims in published maps and institutional affiliations. 